\title{
Conference report: gold highlights at the International Conference on Nanomaterials and Nanotechnology 2011 (ICNANO-2011) in Delhi, India, 18-21 December 2011
}

\author{
Sónia A. C. Carabineiro
}

Published online: 4 May 2012

(C) The Author(s) 2012. This article is published with open access at Springerlink.com

\section{Nano-biomaterials and biomedicals}

The conference started with a plenary lecture from one of the conference chairs, Anthony Turner (Linköping University, Sweden), who spoke about 'Nanomaterials for biosensors and bioelectronics' and showed that several sensors contain gold films or gold surfaces. In fact, in surface plasmon resonance imaging, the sensor surface is almost always a thin layer of gold. Most immobilisation techniques involve the first layer of a chemical linker directly bound to the gold surface, allowing subsequent anchoring of molecules of interest [1]. Gold on glass electrodes are also starting points for the fabrication of artificial enzyme electrodes [2] and Au sputtered layers in the template synthesis of polyaniline nanostructures (Fig. 1) [3]. Turner was the winner of the Acharya Vinoca International award 2011 for his work on biosensors and bioelectronics (Fig. 2). This award is named after Vinayak Narahari Bhave, an Indian advocate of non-violence and human rights, and is given annually by the Vinova Research Institute of India, for notable and outstanding research in Materials Science and Technology.

Ashutosh Tiwari (from the same university) was the winner of the Nano Award 2011 (Fig. 2), for his work on gold nanobioelectronics. This award is honoured annually by the Vinova Bhave Research Institute for notable and outstanding research in the field of Nanoscience and Nanotechnology. Tiwari's plenary lecture dealt with the fabrication on an amperometric biosensor for the quantitative determination of urea in aqueous medium using hematein, a $\mathrm{pH}$ -

\footnotetext{
S. A. C. Carabineiro $(\square)$

Laboratory of Catalysis and Materials, Department of Chemical

Engineering, Faculty of Engineering, University of Porto,

Rua Dr. Roberto Frias, s/n,

4200-465 Porto, Portugal

e-mail: scarabin@fe.up.pt
}

sensitive natural dye [4]. The urease (Urs) was covalently immobilised onto an electrode made of gold nanoparticles functionalized with hyperbranched polyester-Boltron ${ }^{\circledR} \mathrm{H} 40$ ( $\mathrm{H} 40-\mathrm{Au})$ coated onto an indium-tin oxide (ITO)-covered glass substrate (Fig. 3). Chitosan/gold-MPA nanocomposites for sequence-specific oligonucleotide detection were also mentioned. A 20-mer single-stranded oligodeoxyribonucleotide was covalently probed onto the nanocomposite electrode, made up of an ITO glass surface coated with chitosan, which is bonded with carboxyl functionalized thiol capped gold nanoparticles [5]. TEM micrograph of the composite electrode showed that gold nanoparticles had the diameter ranging from 4 to $16 \mathrm{~nm}$.

Robert Gengan (Durban University of Technology, South Africa) spoke about the preparation of novel 3-amino-9ethyl carboazole functionalized gold nanoparticle synthesis. The cytotoxicity of aflatoxin $\mathrm{B}_{1}$, 3-amino-9-ethylcarboazole and gold nanoparticles on A549 cell lines was determined using a bioassay.

Soma Chattopadhyay (Illinois Institute of Technology, USA) spoke about the synthesis and EXAFS study of Au core-Ag shell nanoparticles inside unmodified horse spleen apoferritin protein, with $\mathrm{Au}$ core diameter smaller than $2 \mathrm{~nm}$.

Alok Pandya (Gujarat University, India) talked about a water-soluble glucose biosensor based on an ultrasensitive boronic acid-calix[4]arene functionalized gold nanoprobe. This molecular receptor shows naked eye colour change from pink to blue due to aggregation, as confirmed by other techniques.

Neelam Verma (Punjab University, India) spoke about gold and silver nanoparticles used as sensors for electrochemical detection of organophosphorous pesticides. The lowest detection limit achieved for methyl parathion detection was $0.25 \mathrm{ppb}$, and for chlopyriphos, it was $1 \mathrm{ppt}$. 


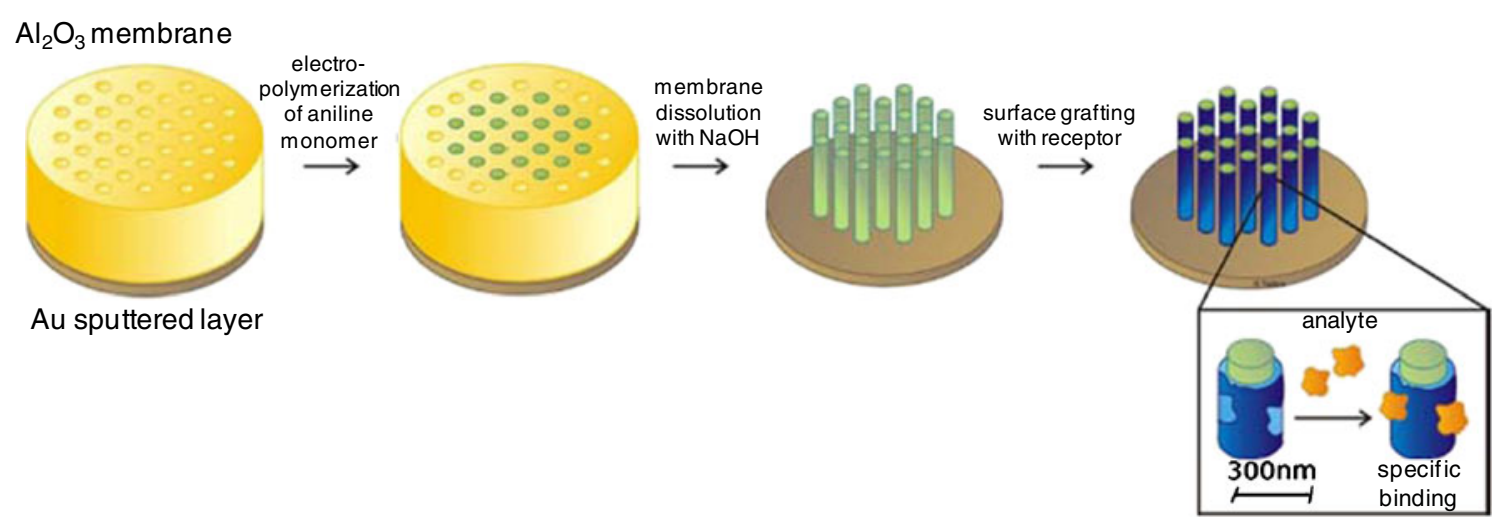

Fig. 1 Scheme of the template synthesis of polyaniline nanostructures (adapted from [3])

Minni Singh's oral presentation (from the same university) was about the enhanced response in electrochemical detection of glucose using model enzyme glucose oxidase by incorporating bimetallic $\mathrm{Au}-\mathrm{Pt}$ nanoparticles in the conducting immobilisation matrix polypyrrole. The improved sensitivity of detection is particularly important for clinical diagnostics, food safety and environmental applications.

Dinesh Kumar (Banasthali University, India) spoke about core-shell $\left(\mathrm{SiO}_{2} @ \mathrm{Ag} / \mathrm{Au}\right)$, metallic $(\mathrm{Ag} / \mathrm{Au})$ and capped metallic (Au/Ag@citrate) nanomaterials used as sensors for heavy metal ions (Cd(II), $\mathrm{Zn}$ (II), Fe(III) and $\mathrm{Pb}(\mathrm{II})$ ) detection in water. These materials showed good performance and were simple, fast and possible to use by non-experts.

C.S. Pundir (Maharshi Dayanand University, India) talked about the immobilisation of oxalate oxidase onto gold nanoparticle-porous $\mathrm{CaCO}_{3}$ microsphere hybrid encapsulated in silica sol and deposited on a Au electrode, for amperometric determination of oxalate in biological fluids (urine, plasma, fruits and vegetables). The biosensor showed to be reliable, have high sensitivity and was able to measure concentrations of oxalate as low as $1 \mu \mathrm{M}$.

Priyanka Sharma (Institute of Microbial Technology, India) presented a poster on the synthesis and characterisation of gold-coated iron oxide core-shell nanoparticles functionalised with receptor molecules (antibodies or proteins) to be used as functional biomaterials. They proved to be viable for exploiting the gold surface protein-binding reactivity for bioassay and the iron oxide core magnetism for magnetic bioseparation.
A. Chopra (from the same institute) presented a poster on a novel low-cost laser-ablated gold sensor modified with multiwalled carbon nanotubes using cysteamine. The presence of nanotubes on the surface of the Au electrode improved the direct transfer of glycated haemoglobin due to its intrinsic activity and a linear reproductible response was obtained. The method presented proved to be selective, sensitive, inexpensive and promising since the presently available tests for this haemoglobin are quite complicated and costly.

Deepika Bhatnaga's poster (Central Scientific Instruments Organization, India) and $N$. Chauhan's poster (Kurukshetra University, India) also reported on the use of multiwalled carbon nanotubes to modify a gold screen-printed electrode for direct electrochemistry and label-free detection of haemoglobin, and for a nanocomposite of Au nanoparticles and polyaniline for improved amperometric determination of lysine, respectively.

Prem Pandey's poster (Banaras Hindu University, India) reported on a prussian blue/gold nanoparticle nanocomposite to be used in the electrocatalytic determination of glutathione, with promising results.

Bindu Sharma's poster (Karnatak University Dharwad, India) dealt with the synthesis and optical characterization of biocompatible fluorescent gold nanoparticles, while Krishnan Anand (Durban University of Technology, South Africa) presented a poster on thiol-dual ligand gold nanoparticles (synthesis and bioassay).

Shobhana K. Menon (Gujarat University, India) presented a poster on a rapid detection of codeine using gold
Fig. 2 Anthony Turner (left) and Ashutosh Tiwari (right) from Linköping University, Sweden, receiving the Acharya Vinoca International Award 2011 and the Nano Award 2011, respectively, for their work on gold biosensors and bioelectronics (see text for details)
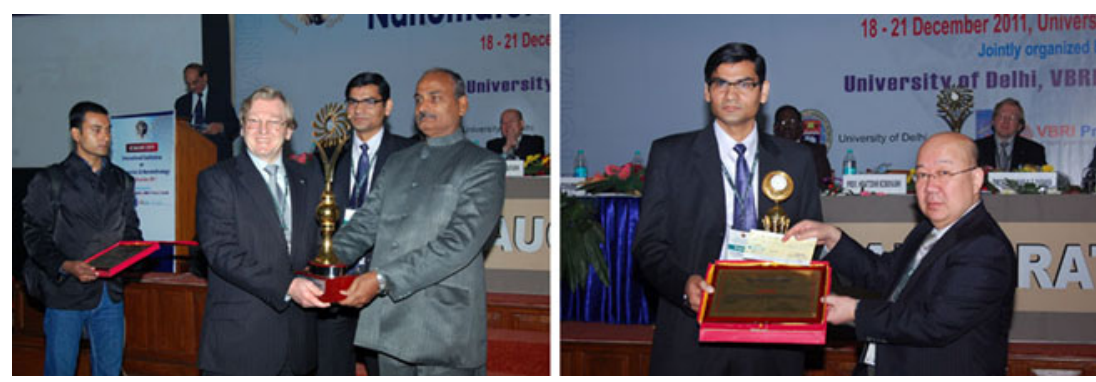
Fig. 3 a Preparation of hyperbranched gold ( $\mathrm{H} 40-\mathrm{Au})$ nanoparticles and $\mathbf{b}$ fabrication of $\mathrm{H} 40-\mathrm{Au} / \mathrm{ITO}$ and Urs/H40-Au/ITO electrodes (adapted from [4])
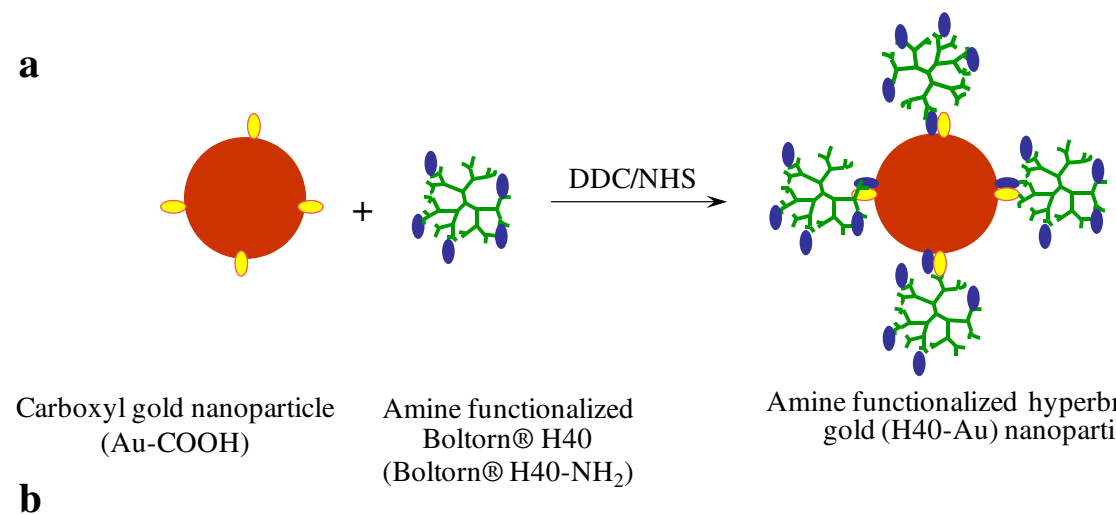

Amine functionalized hyperbranched gold $(\mathrm{H} 40-\mathrm{Au})$ nanoparticle

\section{b}

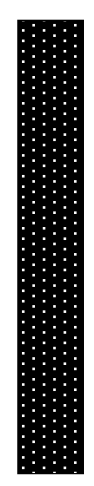

ITO coated glass

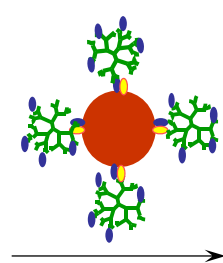

Drop casting

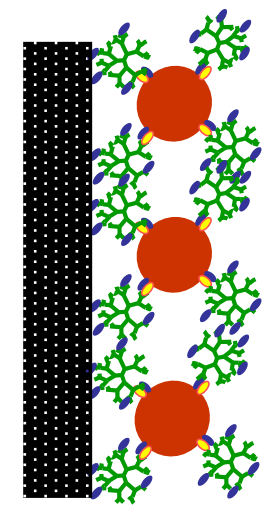

H40-Au/ ITO

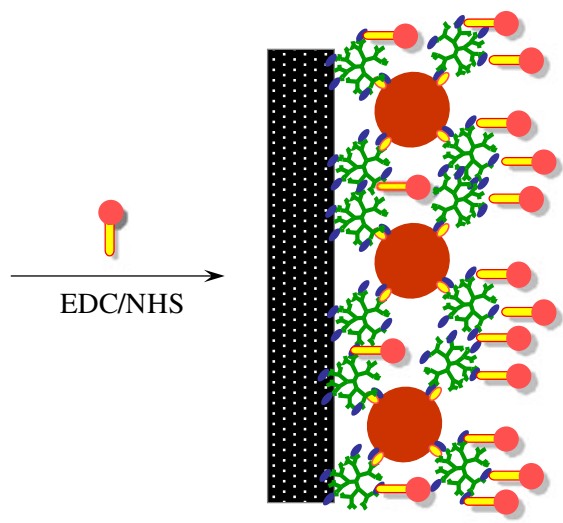

Urs/H40-Au/ ITO

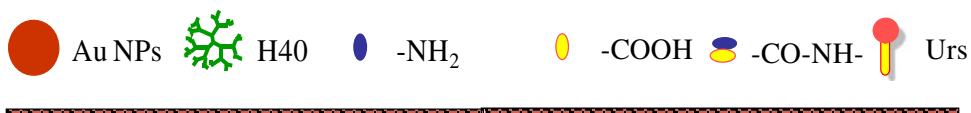

nanoparticles as a probe. In this work, nanoparticles at nanomolar concentration can be clearly observed by the naked eye and allow sensitive detection with minimal consumption of drug samples from a crime scene. This can have interesting applications in forensic science since codeine and heroin drugs (Fig. 4, left and middle) are rapidly metabolised to morphine (Fig. 4, right) and the detection of the latter alone does not provide complete information.

Arghya Bandyopadhyay (University of Kalyani, India) presented a poster on the synthesis of nanofilm assemblies of gold protected by monodispersed iron oxide core metal shell magnetic nanoparticles. This novel nanocomposite may be used to develop a biosensor for detecting biomolecules quantitatively and qualitatively.

S. Malathi (University of Madras, India) presented a poster on the synthesis of gold nanoparticles by a green method using chitosan as a reducing/capping agent. These $\mathrm{Au}$ nanoparticles of $\sim 10 \mathrm{~nm}$ were then used as carriers for the controlled delivery of the antituberculosis drug rifampicin and its catalytic activity was examined for gram-positive and gram-negative bacteria with promising results.

Balu A. Chopade's group (University of Pune, India) presented a poster on the use of Dioscorea bulbifera tuber extract (also called 'air potato', a plant with therapeutic use in Indian and Chinese traditional medicine) mediated synthesis of gold nanoparticles (gold nanotriangles, nanoprisms, nanotrapezoids and nanospheres). These bioreducted gold nanoparticles showed potent anticancer activity against three cancer cells lines, namely HL60, MCF7 and HeLa.

S.K. Pandey (Punjab University, India) presented a poster on gold nanoparticles induced chemiluminescence for VI antigen detection (a virulence factor of typhoid fever caused
Fig. 4 Molecular structures of codeine (left), heroin (middle) and morphine (right) drugs
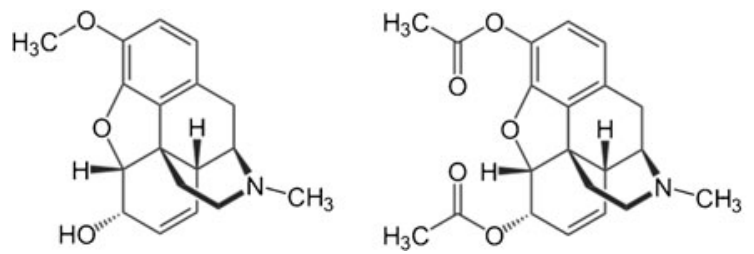

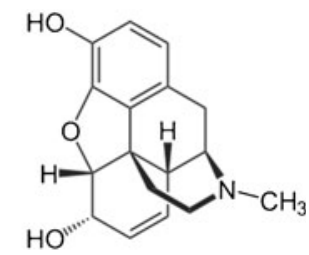


by Salmonella enterica serovar Typhi that is a life-threatening systemic infection and a major public health problem in developing countries). The proposed method is promising for the determination of clinically important bioactive analytes and may prove to be valuable in detecting important virulence strains of pathogens.

Saptarshi Chatterjee (University of Kalyani, India) presented a poster on the successful preparation of glutathionefunctionalised gold nanoparticles used on a novel method of transformation of plasmid DNA in Escherichia coli. The process is less time consuming and increased the transformation efficient when compared to conventional methods. Plus, the Au nanoparticles are non-toxic making this gene delivery method suitable for biotechnological applications.

S. Lata (Maharshi Dayanand University, India) presented a poster dealing with nickel oxide nanoparticles/carboxylated multiwalled carbon nanotubes/polyaniline hybrid films electrodeposited on the surface of a gold electrode. Cytochrome $\mathrm{c}$ oxidised obtained from goat heart was covalently immobilised onto the hybrid film in order to construct an enzyme electrode. A biosensor was further constructed, which gave accurate and satisfactory results for the determination of cytochrome $\mathrm{c}$ on different serum samples.

\section{Nano-fabrication, characterization and properties}

V. N. Bhoraskar's plenary lecture (University of Pune, India) was about the synthesis and modification of $\mathrm{Au}, \mathrm{Ag}, \mathrm{Cu}$, $\mathrm{CdS}$ and Ni nanoparticles by electron irradiating the respective chemical solutions with $6.5 \mathrm{MeV}$ electrons and also the thin films of the respective elements by $15 \mathrm{keV}$ electrons [6]. The average size of the particles could be tailored in the range of 50-130 nm for gold (Fig. 5). It was shown that $\mathrm{Au}$ nanoparticles could diffuse in polymers up to a depth of $\sim 2 \mu \mathrm{m}$, using this method.

Habib Ullah's oral presentation (Pusan National University, Korea) concerned a work carried out in collaboration

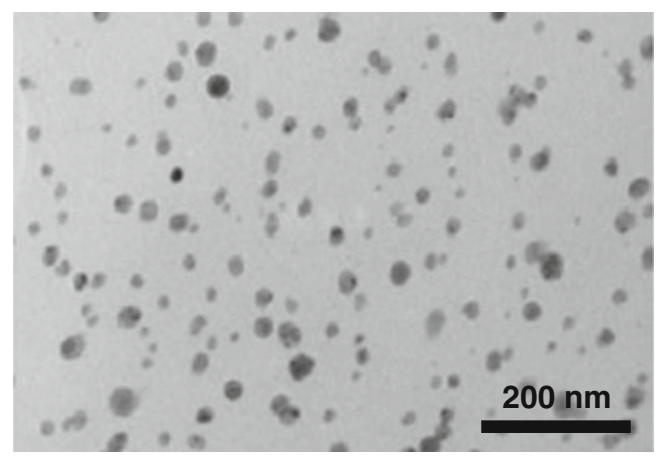

Fig. 5 TEM image of a $15 \mathrm{keV}$ electron-irradiated Au coating (adapted from [6]) with the American International University of Bangladesh, dealing with water-soluble gold nanoparticles coordinated to poly(vinylpyrrolidone) (PVP). The final gold colloids were very stable as the PVP molecules coordinated through the $\mathrm{C}-\mathrm{N}$ and $\mathrm{C}=\mathrm{O}$ sites, instead of the $\mathrm{C}=\mathrm{O}$ site alone.

Ali Ayati's oral presentation (Ferdowsi University of Mashhad, Iran) concerned the synthesis of stabilised $45 \mathrm{~nm}$ gold nanorods generated by a green chemistry type process using $\mathrm{H}_{6}\left[\mathrm{PMo}_{9} \mathrm{~V}_{3} \mathrm{O}_{40}\right]$, a harmless polyoxometalate (POM), under UV irradiation and in the absence of any surfactant or seed. This POM plays the role of a photocatalyst, reducing agent and efficient stabiliser.

Biswajit Chowdhury (Indian School of Mines, India) described the preparation of ceria-based mixed oxide solid solutions having nanocrystallinity, where not only the pore size but also the defect sites can be created in the mixed oxide by doping lower valent cations. The gold nanoclusters on the oxide surface can dissociate the chemisorbed oxygen which diffuses through the defect oxides, providing excellent activity towards benzyl alcohol oxidation using molecular oxygen.

Sajid Ali Ansari (Aligarh Muslim University, India) presented a poster on polyaniline silver and gold composites prepared by in situ polymerization of aniline and ammonium peroxydisulphate as oxidising agent and $\mathrm{Ag}$ and $\mathrm{Au}$ colloidal nanoparticle solutions (reduced with sodium borohydride). These composites showed to have good optical and dielectric properties.

S. Tripathi (UGC-DAE Consortium for Scientific Research, India) in a collaborative work with the Karlsruher Institut für Technologie in Germany and the Catholic University of Leuven, Belgium, presented a poster with results of structural and magnetic characterisation of $\mathrm{Co} / \mathrm{Au}$ system with two different compositions where Co was grown as a discontinuous layer sandwiched between a pair of Au layers. Interesting magnetic and structural transformations of the layers were observed due to the formation of a non-magnetic phase at the interface with temperature increase.

Parul Khurana (Banasthali University, India) had a poster on the synthesis and plasmonic properties of $\mathrm{Au}-\mathrm{Ag}$ core-shell nanostructures. Ag and $\mathrm{Au}$ nanoshells were grown on silica microspheres with diameters ranging from 50 to $60 \mathrm{~nm}$. Reduction of $\mathrm{Au}$ and $\mathrm{Ag}$ precursors onto gold decorated microspheres resulted in increasing gold coverage on the silica core. The product may also have many potential applications in optical, magnetic, biochemical and biomedical fields.

Sheenam Thatai (from the same group) had a poster dealing with the detection of $\mathrm{Cd}^{2+}, \mathrm{Zn}^{2+}$ and $\mathrm{Pb}^{2+}$ ions in water using Au@citrate and $\mathrm{SiO}_{2} @ \mathrm{Au}$ nanoparticles as highly selective and sensitive nanosensors. The presence of metal ions changes the colour of the solution, visible to the naked eye, and causes aggregation of nanoparticles. 
F. Hashemi (Khayyam University, Iran) presented a poster on theoretical investigations of the substituent effect on the molecular wire, $\mathrm{Au} / \mathrm{guanine} / \mathrm{Au}$, performed using density functional calculations by considering the influence of an external electric field. The obtained results showed that an organic molecule can be functionalised as a molecular diode.

\section{Nano-advanced materials}

Palani Barathi (Velore Institute of Technology University, India) spoke about a nickel hexcyanoferrate-modified disposable gold nail electrode for electrocatalytic response of hydrazine in neutral $\mathrm{pH}$. The sodium sulphate-sodium acetate $\mathrm{pH} 7$ solution showed a well-defined, reversible and stable voltammetric responses and enhanced catalytic activity towards hydrazine without any excess alkali metal cation added solution. Under optimal conditions, the modified electrode showed a linear response for hydrazine in the concentration range from 0.5 to $10 \mathrm{mM}$.

S.K. Boruah (Gauhati University, India) presented a poster dealing with green synthesis of gold nanoparticles using a simple, fast, low-cost and economical technique, using fresh leaves and buds of Camellia sinensis tea (Fig. 6). The polyphenols present in the young leaves of tea extract reduce $\mathrm{HAuCl}_{4}$ at room temperature. The core size of gold nanoparticles decreases as the amount of tea extract increases. Particles ranging from 9 to $12 \mathrm{~nm}$ were obtained.

The poster of M. Yazdani (Shiraz University, Iran) was about the synthesis of gold nanoparticles with imidazoliumbased ionic liquids, which show preferential binding affinity towards gold crystal surfaces. 1-Dodecyl-3-methyl imidazolium tryptophan (long chain) and 1-ethyl-3-mehyl imidazolium tryptophan (short chain) were used, in order to test the effect of steric repulsion on the gold nanoparticles stability, being shown that the longer chain ionic liquids produced better results. Those nanoparticles showed to have a hydrophilic nature.

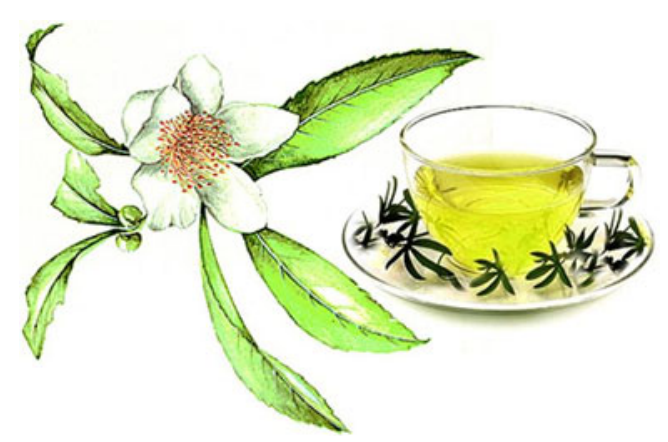

Fig. 6 C. sinensis leaves and flower used in tea
B. Batra (Maharshi Dayanand University, India) presented a poster on immobilisation of tyramine oxidase (purified from black gram seeds) on citrate-coated silver nano particles, deposited on a Au electrode, through cysteine layer, to obtain a tyramide biosensor that showed good performance in the detection of tyramide in beer and sauce, with a detection limit of $0.01 \mathrm{mM}$. Its advantage is that it does not suffer from leaching of the enzyme and measures this compound specifically.

A. Kedia (University of Delhi, India) presented a poster describing a simple, versatile and environmentally friendly one-step room temperature chemical synthesis route for the preparation of polyvinyl pyrrolidone functionalised size/ shape-controlled gold nanoparticles by in situ polymerisation of the $\mathrm{N}$-vinyl pyrrolidone monometer using $\mathrm{HAuCl}_{4}$ as the oxidant. Water molecules act as a nucleophile that attacks the gold-vinyl complexes.

Ida Tiwari (Banaras Hindu University, India) presented a poster dealing with the addition of gold nanoparticles to multiwalled carbon nanotubes which were used to modify a glassy carbon electrode that showed good electrocatalytic activity towards the oxidation of NADH enzyme, better adhesion properties, good stability and no leaching of nanocomposite, thus being a promising electrochemical sensor for this enzyme in the future.

A collaborative work of Naheed Ahmad et al. (Patna University, India) and the Universities of Magadh, India, and of Aveiro, Portugal, dealt with the rapid green synthesis of silver and gold nanoparticles from the biowaste of pomegranate fruit (Punica granatum) at room temperature. The extracts of all parts of the fruit have therapeutic properties and the biosynthetic products along with reduced cofactors play an important role in the reduction of the $\mathrm{Au}$ salt to nanoparticles of $\sim 10 \mathrm{~nm}$, which showed to be quite stable.

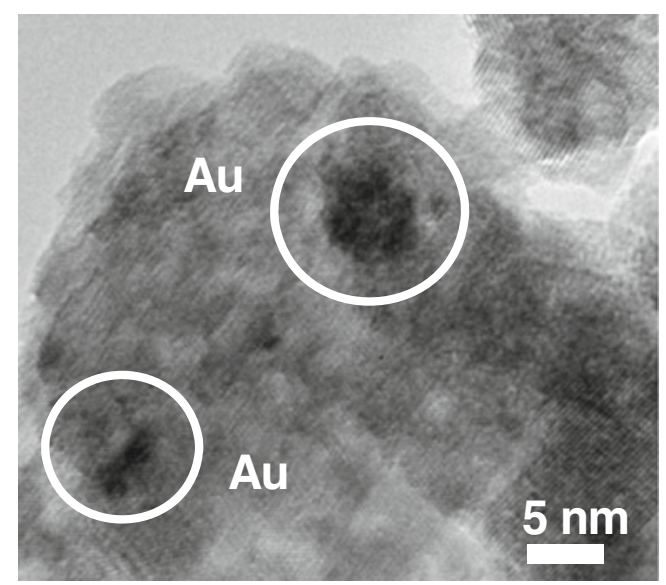

Fig. 7 HRTEM image of a Ce-Mn-O support (with a molar percentage of $30 \% \mathrm{Mn}$ and $70 \% \mathrm{Ce}$ ) with gold nanoparticles (adapted from [7]) 
Sónia Carabineiro (University of Porto, Portugal) presented two posters dealing with gold nanoparticles supported on oxides, used for $\mathrm{CO}$ oxidation, namely, $\mathrm{Ce}-\mathrm{Mn}-\mathrm{O}$ composite materials. It was shown that the addition of $\mathrm{Ce}$ to $\mathrm{Mn}_{x} \mathrm{O}_{y}$ produced supports with larger surface area, which led to smaller gold nanoparticles (Fig. 7) and consequentially improved catalytic activity. Another poster dealt with the heterogenation of organometallic complexes of several metals on carbon materials, to be used as catalysts for alkane oxidation. Heterogenised gold complexes showed promising results.

Deepshikha (Amity University, India) had a poster on the synthesis of polymer nanocomposites based on nanostructured polyaniline, gold nanoparticles and graphene nanosheets, by in situ polymerization. The nanodispersion could also be electrodeposited to produce a uniform nanofilm on an indium tin oxide surface. These composites showed good electrochemical properties and conductivity.

M. Misra's poster (Central Scientific Instruments Organization, India) also dealt with indium tin oxide, used as substrate for gold nanoparticles caped $\mathrm{TiO}_{2}$ nanorod arrays for use in dye-sensitised solar cell technology. The $\mathrm{Au}$ nanoparticles on $\mathrm{TiO}_{2}$ strongly absorb light, due to the localised surface plasmon resonance, and thereby promote light absorption of the dye.

J.P. Singha (King Saud University, Saudi Arabia) presented a poster dealing with the immobilisation of $\mathrm{Pt}, \mathrm{Au}$ and $\mathrm{Pt}-\mathrm{Au}$ nanoparticles on highly ordered $\mathrm{TiO}_{2}$ nanotubes by pulse electro-deposition. The electrochemical behaviour of these composites was examined through the study of oxygen reduction.

Isha Mudahar (Khalsa College, India) presented a poster dealing with density functional calculations on pure dimers $\left(\mathrm{C}_{60}-\mathrm{C}_{60}\right)$ and dimers doped with $\mathrm{N}$ and $\mathrm{B}\left(\mathrm{C}_{60-x} \mathrm{~N}_{x}-\mathrm{C}_{60-}\right.$ ${ }_{x} \mathrm{~B}_{x}$ ) with gold contacts, in order to investigate the charge transfer in molecular nano junctions across $\mathrm{C}_{60}$ dimers. The results obtained suggest that there is a charge transfer from $\mathrm{C}$ to $\mathrm{Au}$ atoms.
Acknowledgments I am grateful to Prof. Anthony Turner for providing me the slides of his plenary lecture. Fundação para a Ciência e Tecnologia (FCT) is acknowledged for funding (CIENCIA 2007 programme and project PTDC/QUI-QUI/100682/2008, financed by FCT and FEDER in the context of Programme COMPETE).

Open Access This article is distributed under the terms of the Creative Commons Attribution License which permits any use, distribution and reproduction in any medium, provided the original author(s) and source are credited.

\section{References}

1. Scarano S, Mascini M, Turner APF, Minunni M (2010) Review: surface plasmon resonance imaging for affinity-based biosensors. Biosens Bioelectron 25:957-966

2. Lakshmi D, Withcombe MJ, Davis F, Chianella I, Piletska EV, Guerreiro A, Subrahmanyam S, Brito PS, Fowler SA, Piletsky SA (2009) Chimeric polymers formed from a monomer capable of free radical, oxidative and electrochemical polymerisation. Chem Commun 9:2759-2761

3. Berti F, Todros S, Lakshmi D, Chianella I, Ferroni M, Piletsky SA, Turner APF, Marrazza G (2010) Quasi-monodimensional polyaniline nanostructures for enhanced molecularly imprinted polymerbased sensing. Biosens Bioelectron 26:497-503

4. Tiwari A, Aryal S, Pilla S, Gonga S (2009) An amperometric urea biosensor based on covalently immobilized urease on an electrode made of hyperbranched polyester functionalized gold nanoparticles. Talanta 78:1401-1407

5. Cao S, Mishra R, Pilla S, Tripathi S, Pandey MK, Shah G, Mishra AK, Prabaharan M, Mishra SB, Xin J, Pandey RR, Wu W, Pandey AC, Tiwari A (2010) Novel chitosan/gold-MPA nanocomposite for sequence-specific oligonucleotide detection. Carbohydr Polym 82:189-194

6. Mahapatra SK, Bogle KA, Dhole SD, Bhoraskar VN (2007) Synthesis of gold and silver nanoparticles by electron irradiation at 5-15 keV energy. Nanotechnology 18(13):135602

7. Carabineiro SAC, Silva AMT, Dražić G, Tavares PB, Figueiredo JL (2012) CO oxidation using gold supported on Ce-Mn-O composite materials. In: DiLoreto D, Corcoran I (eds) Carbon monoxide: sources, uses and hazards. Nova Publishers, Nova Science Pub Inc., New York 